\title{
Shuxuetong injection simultaneously ameliorates dexamethasone-driven vascular calcification and osteoporosis
}

\author{
ZHE XU $^{1 *}$, XIAOGUANG LIU ${ }^{1 *}$, YANQING LI ${ }^{2}$, HONGLIANG GAO ${ }^{1}$, \\ TAO HE ${ }^{1}, \mathrm{CHUNLEI} \mathrm{ZHANG}^{1}$, WEI HAO ${ }^{1}$ and $\mathrm{XU} \mathrm{TENG}^{3,4}$

\begin{abstract}
Departments of ${ }^{1}$ Anesthesiology and ${ }^{2}$ Gynecology, Hebei Provincial Hospital of Traditional Chinese Medicine, Shijiazhuang, Hebei 050011; ${ }^{3}$ Department of Physiology, Hebei Medical University, Shijiazhuang, Hebei 050017;
\end{abstract} \\ ${ }^{4}$ Hebei Collaborative Innovation Center for Cardio-Cerebrovascular Disease, Shijiazhuang, Hebei 050000, P.R. China
}

Received May 14, 2020; Accepted November 12, 2020

DOI: $10.3892 /$ etm.2021.9630

\begin{abstract}
Osteoporosis (OP) and vascular calcification (VC) share a number of common risk factors, pathophysiological mechanisms and etiology, which are known as bone-vascular axis. The present study aimed to investigate the effects of Shuxuetong (SXT) injection on VC and osteoporosis. A rat model of VC and osteoporosis was induced by dexamethasone (DEX; $1 \mathrm{mg} / \mathrm{kg} /$ day for 4 weeks, intramuscularly). Simultaneously, $0.6 \mathrm{ml} / \mathrm{kg} /$ day SXT was intraperitoneally injected. Compared with control rats, DEX induced significantly more VC and OP, as determined by increased calcium deposition and alkaline phosphatase activity in the aorta, disturbed structure, decreased levels of cortical bone thickness and trabecular bone area, and increased apoptosis in the bone. SXT injection ameliorated DEX-induced VC and osteoporosis; furthermore, the osteoblastic differentiation of vascular smooth muscle cells and the activation of endoplasmic reticulum stress in the DEX group was also prevented by SXT injection. Compared with control rats, protein expression levels of sclerostin, a crucial crosslink of the bone-vascular axis, were significantly increased in the aorta and bone of rats with DEX, which was also attenuated by SXT injection. Thus, the present study suggested that SXT injection could ameliorate both VC and OP, and may be mediated by the regulation of sclerostin. The present study may provide the basis a novel strategy for the prevention and
\end{abstract}

Correspondence to: Dr Wei Hao, Department of Anesthesiology, Hebei Provincial Hospital of Traditional Chinese Medicine, 389 Zhongshan East Road, Shijiazhuang, Hebei 050011, P.R. China E-mail: Hao_wei800@163.com

Dr Xu Teng, Department of Physiology, Hebei Medical University, 361 Zhongshan East Road, Shijiazhuang, Hebei 050017, P.R. China E-mail: tengxu78@163.com

*Contributed equally

Key words: Chinese traditional medicine, bone-vascular axis, glucocorticoid, sclerostin, endoplasmic reticulum stress treatment of VC and OP, which emerge as side-effects of glucocorticoids.

\section{Introduction}

Osteoporosis (OP) and vascular calcification (VC) are known worldwide as major risk factors of mortality and morbidity $(1,2)$. VC causes alterations to the blood pressure profile and an increased pulsatile pressure and flow load, which can damage target organs, such as the heart, brain and kidneys, and therefore increases the risk of cardiovascular morbidity and mortality (3). OP has been indicated to result in increased susceptibility to fracture and painful morbidity (4). Although both factors were considered independently for a long time, studies have suggested a close relationship between $\mathrm{OP}$ and VC $(1,2)$. Both OP and VC share a number of common risk factors, pathophysiologic mechanisms and etiology $(5,6)$, which is well summarized in the expression 'bone-vascular axis' that was coined to describe the cross-talk between bone and cardiovascular metabolism (7). Several emerging findings have suggested that bone loss may promote $\mathrm{VC}$, and that $\mathrm{VC}$ also has an impact on bone metabolism $(8,9)$; therefore, it is of crucial importance to establish a treatment that targets both disorders.

Although associations between $\mathrm{VC}$ and bone loss have been demonstrated (3), it remains unclear which exact mechanisms are core in the bone-vascular axis. VC and osteoporosis share a number of risk factors, including aging, chronic sterile inflammation and metabolic disorders (3). However, considerable research is required to identify the mechanisms that link VC to bone deterioration. Several molecules have been reported to serve a crucial role in the bone-vascular axis (9), among which, sclerostin might serve a major role (10-13). Sclerostin, a 22-kDa glycoprotein, is a well-known inhibitor of bone formation (14). However, previous studies have also reported the potential role of sclerostin in the development of VC (14-16). Sclerostin positively correlates with the presence of atherosclerosis, and is present in atherosclerotic plaques, mainly expressed by vascular smooth muscle cells (VSMCs) in patients $(14,15)$. Furthermore, the expression of sclerostin in calcifying VSMC has been observed, and it has been demonstrated to have a protective role in the vasculature (16). 
These results suggest that sclerostin may be a target for the simultaneous regulation of $\mathrm{VC}$ and osteoporosis.

Injection of Shuxuetong (SXT), a Chinese Materia Medica standardized product extracted from Hirudo and Pheretima, has been widely used in Traditional Chinese Medicine, particularly in patients with stroke and myocardial infarction (17-19). Each ampoule of SXT $(2 \mathrm{ml})$ is derived from $\sim 0.5 \mathrm{~g}$ leech and $\sim 0.5 \mathrm{~g}$ earthworm (18), and the main active ingredients are considered to be peptides, glycopeptides and oligosaccharides (20). In addition to its antithrombotic, anticoagulant and fibrinolytic activities (21), injection of SXT injection has also been shown to confer vascular protection $(18,20)$. In addition, SXT has been reported to decrease the levels of pro-inflammatory cytokines and inhibit the inflammation (22-24) that contributes to progression of VC and osteoporosis (5). Therefore, it was hypothesized that the injection of SXT could be used for the simultaneous treatment of VC and osteoporosis by regulating the vascular-bone axis.

Epidemiologic research demonstrated that glucocorticoids (GCs) have long been used globally for inflammatory, immunologic and allergic disorders (25). However, long-term exposure to GCs simultaneously induces osteoporosis and VC (26-29). The synthetic long-acting GC dexamethasone (DEX) may cause more severe adverse effects compared with short-acting medication such as methylprednisolone (26). DEX may induce VC by downregulating calcification-inhibiting molecules and accelerating osteoblastic differentiation of VSMCs $(27,28)$, while osteonecrosis of the femoral head could also be caused by DEX via activation of endoplasmic reticulum stress (ERS) (29). Thus, a rat model of simultaneous VC and OP induced by DEX was used in the present study to investigate the ameliorative effect of SXT injections.

\section{Materials and methods}

Experimental protocols. All animal care and experimental protocols were in compliance with the P.R. China Animal Management Rule (30) and the National Institutes of Health Guide for the Care and Use of Laboratory Animals (31), and were approved by the Animal Care Committee of the Hebei Provincial Hospital of Chinese Medicine (Shijiazhuang, China; approval no. 2019-KY-012-01). Male Sprague-Dawley (SD) rats ( 8 weeks old; $200-220 \mathrm{~g}$ ) were supplied by the Animal Center of the Hebei Medical University (Hebei, China). All animals were housed at $22 \pm 2^{\circ} \mathrm{C}$ with a relative humidity of $50 \pm 10 \%$ and a 12 -h light/dark cycle. The animals had free access to water and food.

A total of 32 rats were randomly divided into four groups: i) Control group (Con), only treated with vehicle; ii) SXT group, intraperitoneally injected with $0.6 \mathrm{ml} / \mathrm{kg} / \mathrm{day}(19,20)$ SXT (Mudanjiang Youbo Pharmaceutical Co. Ltd.) for 4 weeks; iii) VC and osteoporosis group (DEX), intramuscularly injected with $1 \mathrm{mg} / \mathrm{kg} /$ day DEX (Guangzhou Baiyunshan Tianxin Pharmaceutical Co., Ltd.) for 4 weeks; iv) DEX plus SXT injection group (DEX + SXT). The recommended dose of $\mathrm{SXT}$ injection in the manufacturer's package insert is $0.1 \mathrm{ml} / \mathrm{kg} / \mathrm{day}$. The coefficient of conversion between human $(60 \mathrm{~kg})$ and rats $(200 \mathrm{~g})$ is $\sim 6$. Therefore, the dose of $0.6 \mathrm{ml} / \mathrm{kg} / \mathrm{day}$ was used in the present study. After 4 weeks of treatment, the rats were anesthetized with $50 \mathrm{mg} / \mathrm{kg}$ sodium pentobarbital, and then sacrificed for further assessment.

Histological staining. Thoracic aortas were fixed in $10 \%$ neutral buffered formaldehyde for $24 \mathrm{~h}$ at $4^{\circ} \mathrm{C}$, embedded in paraffin, cut into $6-\mu \mathrm{m}$-thick sections, and stained with Alizarin Red S at room temperature to measure the amount of calcium deposition in the vessels. The two femoral heads were harvested through dissection and placed in $10 \%$ neutral buffered formaldehyde for $72 \mathrm{~h}$ at $4^{\circ} \mathrm{C}$ for fixation. After fixation, the femoral heads were decalcified and stained with hematoxylin and eosin (H\&E) staining. The sections were stained with hematoxylin for $10 \mathrm{~min}$ at room temperature, and after washing with distilled water, they were stained with eosin for $3 \mathrm{~min}$ at room temperature. The slides were visualized using a digital light microscope (magnification, x200; Olympus Corporation).

Immunohistochemistry. For immunohistochemistry, the left femoral heads were harvested through dissection and placed in $10 \%$ neutral buffered formaldehyde for $72 \mathrm{~h}$ at $4^{\circ} \mathrm{C}$ for fixation. The tissues were embedded in paraffin and cut into $6-\mu \mathrm{m}$ thick sections. The sections were then incubated with primary antibodies (Table I) at $4^{\circ} \mathrm{C}$ overnight. Bovine serum albumin (1\%; Sigma-Aldrich; Merck KGaA) was used as the blocking agent at room temperature for $1 \mathrm{~h}$. The primary antibodies were detected after incubation with secondary antibody (Table I) conjugated to horseradish peroxide for $30 \mathrm{~min}$ at $37^{\circ} \mathrm{C}$, and visualized with 3,3'-diaminobenzidine tetrahydrochloride. The slides were visualized using a digital light microscope (Olympus Corporation), and images were analyzed using ImageJ software (v1.48; National Institutes of Health). The positive rate of sclerostin was defined using the following formula: (Positive area/specimen area) $\mathrm{x} 100$.

Terminal deoxynucleotidyl transferase-mediated dUTP nick-end labeling (TUNEL) staining. TUNEL staining was performed for in situ detection of late apoptosis. The bilateral femora were harvested through dissection and placed in $10 \%$ neutral buffered formaldehyde for $72 \mathrm{~h}$ at $4^{\circ} \mathrm{C}$ for fixation. The femora were embedded in paraffin and cut into $6-\mu \mathrm{m}$ thick sections. The sections underwent TUNEL staining using commercial kits according to the manufacturer's instructions (cat. no. C1098; Beyotime Institute of Biotechnology).

Quantification of calcium and alkaline phosphatase (ALP) activity. For the determination of calcium content, aortic tissues were dried at $55^{\circ} \mathrm{C}$, and dissolved in $0.6 \mathrm{~N} \mathrm{HCl}$ at $4^{\circ} \mathrm{C}$ for $24 \mathrm{~h}$. The supernatant fluid was used to measure calcium content by colorimetry in a reaction with o-Cresolphtalein complexone (cat. no. C004-2; Nanjing Jiancheng Bioengineering Institute Co., Ltd.). ALP activity in the aortic vessels was measured using an ALP colorimetric assay kit according to the manufacturer's instructions (cat. no. A059-2-2; Nanjing Jiancheng Bioengineering Institute).

Western blotting. Aortic tissues were homogenized in a lysis buffer [1\% NP-40, $20 \mathrm{mmol} / \mathrm{l}$ Tris-HCl (pH 8.0), 
Table I. Antibody information.

\begin{tabular}{lllc}
\hline Antibody & Cat. no. & & Supplier \\
\hline GRP78 & ab21685 & Abcam & Dilution \\
CHOP & ab10444 & Abcam & $1: 2,000$ \\
Phosphorylated Akt & 4534 & Cell Signaling Technology, Inc. & $1: 500$ \\
Akt & 4691 & Cell Signaling Technology, Inc. & $1: 2,000$ \\
Sclerostin & ab85799 & Abcam & $1: 1,000$ \\
Calponin & ab203047 & Abcam & $1: 1,000$ \\
SM22 $\alpha$ & ab14106 & Abcam & $1: 2,000$ \\
RUNX2 & ab114133 & Abcam & $1: 1,000$ \\
BMP & ab14933 & Abcam & $1: 1,000$ \\
$\beta$-actin & GTX109639 & GeneTex, Inc. & $1: 2,000$ \\
Goat anti-rabbit secondary & GTX213110 & GeneTex, Inc. & $1: 5,000-10,000$ \\
antibody (HRP conjugate) & & &
\end{tabular}

GRP78, glucose-regulated protein 78; CHOP, C/EBP-homologous protein; pAkt, phosphorylated Akt; SM22 $\alpha$, smooth muscle22 $\alpha$; RUNX2, runt-related transcription factor 2; BMP2, bone morphogenetic protein-2; HRP, horseradish peroxidase.

$137.5 \mathrm{mmol} / 1 \mathrm{NaCl}, 1 \mathrm{mmol} / 1 \mathrm{Na}_{3} \mathrm{VO}_{4}, 1 \mathrm{mmol} / 1 \mathrm{PMSF}$ and $10 \mu \mathrm{g} / \mathrm{ml}$ aprotinin]. The lysate's protein concentration was determined using the Bradford method. An equal volume of 2X SDS sample buffer [0.125 mol/1 Tris- $\mathrm{HCl}$ ( $\mathrm{pH} 7.4), 4 \%$ SDS and $20 \%$ glycerol] was added, then the samples were boiled for $5 \mathrm{~min}$. The samples comprising $50 \mu \mathrm{g}$ of protein underwent $10 \%$ SDS-PAGE for $3 \mathrm{~h}$ at $60 \mathrm{~mA}$. The proteins were then electrophoretically transferred to a nitrocellulose membrane and incubated for $1 \mathrm{~h}$ in TBS containing 5\% non-fat powdered milk at room temperature. The membranes were then incubated with the primary antibodies (Table I) at $4^{\circ} \mathrm{C}$ overnight. After washing each membrane with TBS-0.1\% Tween-20 (TBST) three times for $10 \mathrm{~min}$, the membranes were incubated with the secondary antibody (Table I) for $1 \mathrm{~h}$ at room temperature. The membranes were then washed with TBST three times for $10 \mathrm{~min}$ each and detected using enhanced chemiluminescence (cat. no. P1050-500; Applygen Technologies, Inc.). Autoradiographs were scanned, and the relative densities were semi-quantified by ImageJ software (v1.48; National Institutes of Health).

Statistical analysis. Continuous data are presented as the mean $\pm \mathrm{SD}(\mathrm{n}=8 /$ group). Unpaired Student's t-test was performed to compare the results of the two groups. One-way analysis of variance followed by Tukey's post hoc test was performed to compare the results of $>2$ groups. $\mathrm{P}<0.05$ was considered to indicate a statistically significant difference.

\section{Results}

Ameliorative effect of SXT on DEX-induced VC. Alizarin red staining revealed increased calcium deposition, and destruction of elastic fibers and VSMCs in the aortic tunica media of DEX rats, which was notably ameliorated by treatment with SXT (Fig. 1A). Additionally, the DEX-induced increase in calcium and ALP activity in the aorta was also attenuated by injection of SXT (Fig. 1B and C). SXT injection alone did not significantly influence calcium content and ALP activity.
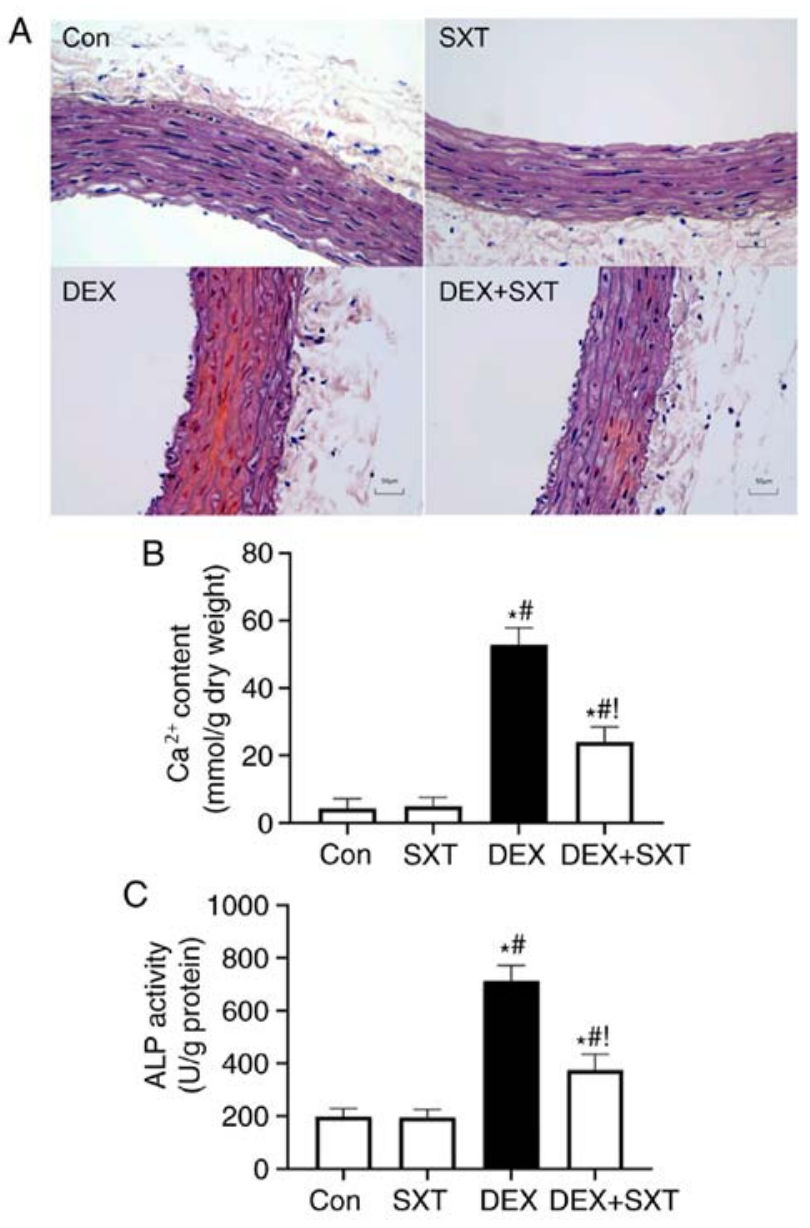

Figure 1. SXT injection ameliorates DEX-induced vascular calcification in rats. (A) Alizarin red staining of the aorta. (B) Calcium content and (C) alkaline phosphatase activity in the aorta. $\mathrm{N}=8$ /group. The data are presented as the mean \pm SD. ${ }^{*} \mathrm{P}<0.05$ vs. Con; ${ }^{*} \mathrm{P}<0.05$ vs. SXT; $\mathrm{P}<0.05$ vs. DEX. DEX, dexamethasone; Con, control; SXT; shuxuetong; ALP, alkaline phosphatase.

The protein expression levels of contractile phenotype markers of VSMCs, including calponin and smooth 


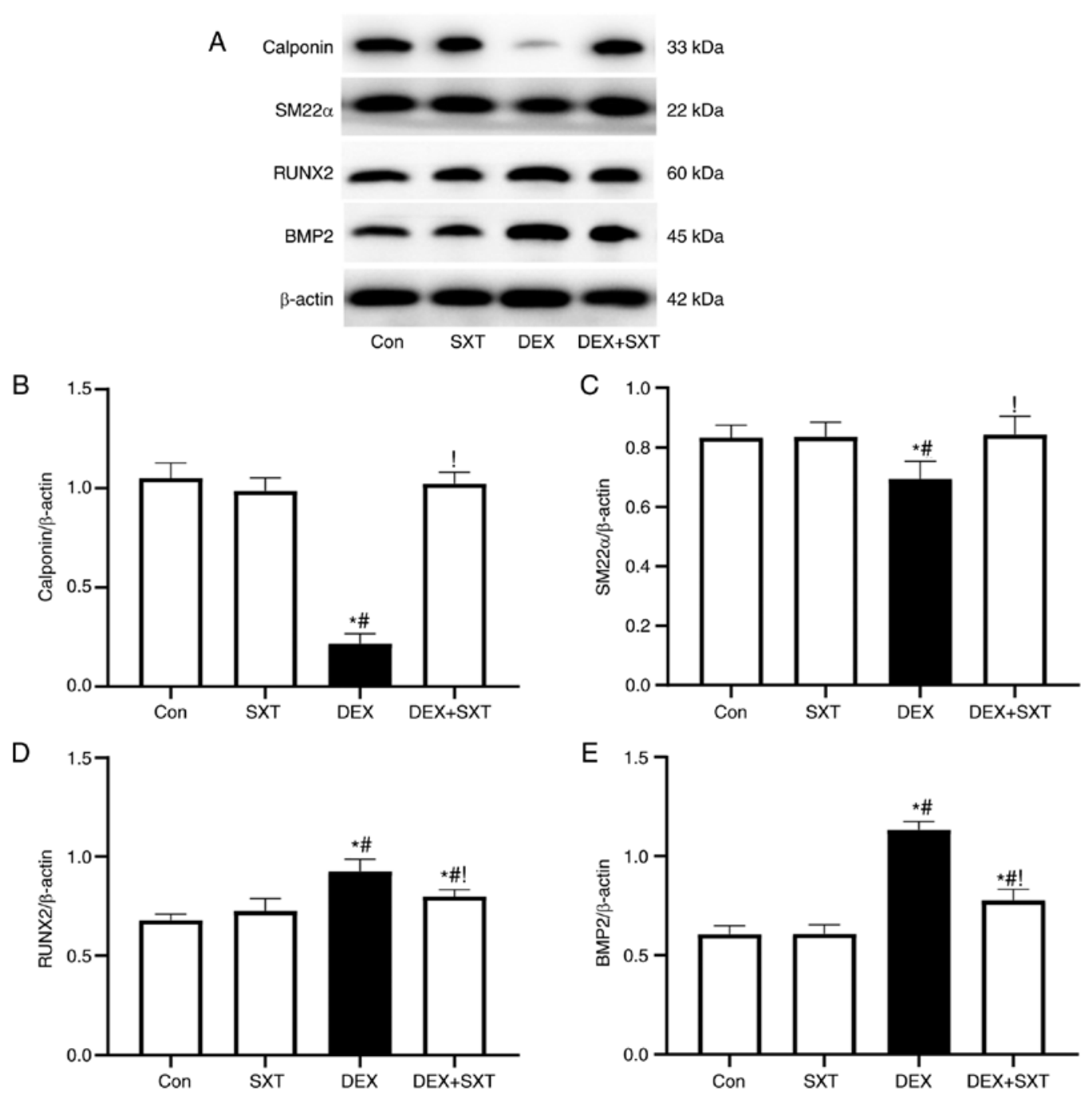

Figure 2. SXT reverses the osteoblastic-like transformation of vascular smooth muscle cells in rats with vascular calcification. (A) Representative western blotting image. (B) Calponin, (C) SM22 $\alpha$, (D) RUNX2 and (E) BMP2 protein expression levels detected by western blotting. N=8/group. The data are presented as the mean \pm SD. ${ }^{~} \mathrm{P}<0.05$ vs. Con; ${ }^{\prime} \mathrm{P}<0.05$ vs. SXT; 'P $<0.05$ vs. DEX. DEX, dexamethasone; Con, control; SXT; shuxuetong; SM $22 \alpha$, smooth muscle $22 \alpha$; RUNX2, runt-related transcription factor 2; BMP2, bone morphogenetic protein-2.

muscle $22 \alpha(\operatorname{SM} 22 \alpha)$, were significantly reduced in the DEX-treated group compared with in the control group, but were restored following injection of SXT (Fig. 2). Injection of SXT also attenuated the increased protein expression levels of the osteoblastic phenotype markers runt-related transcription factor 2 (RUNX2) and bone morphogenetic protein-2 (BMP2) in rats with DEX, whereas the protein expression levels of these markers in the aorta were not significantly influenced in the SXT alone group (Fig. 2).

SXT inhibits DEX-induced activation of ERS and increased levels of sclerostin in the aorta. The protein expression levels of ERS markers, including glucose-regulated protein 78 (GRP78) and C/EBP-homologous protein (CHOP), were determined by western blotting. Compared with the Con and SXT groups, the protein expression levels of GRP78 and CHOP in aortic tissue were significantly increased in the DEX group, and were attenuated in the DEX + SXT group (Fig. 3A-C). Conversely, SXT injection rescued the decrease in relative levels of phosphorylated (p)Akt induced by DEX treatment (Fig. 3D). The increased protein expression levels of sclerostin induced by
DEX in the aorta were also significantly attenuated by treatment with SXT injection (Fig. 4).

SXT ameliorates DEX-induced osteoporosis and apoptosis of the femoral head. H\&E staining demonstrated the destroyed structure of the femoral head in rats treated with DEX, which was markedly ameliorated by injection with SXT (Fig. 5A-D). In line with these structural changes, decreases in cortical bone thickness and trabecular bone area percentage were also significantly ameliorated by SXT treatment (Fig. 5E and F). There was no clear effect of SXT treatment alone on the structure of the femoral head (Fig. 5A-D). Similarly, the apoptotic cell counts determined by TUNEL staining were significantly increased in the DEX group, and SXT injection attenuated this increase in cellular apoptosis (Fig. 6A-E). Furthermore, the results suggested that there was no effect of SXT treatment alone on apoptosis (Fig. 6A-E). Accordingly, the protein expression levels of CHOP, a common marker of ERS that mediates ERS-associated apoptosis (32), were significantly increased in the DEX group and rescued by SXT injection (Fig. 6F-J). 

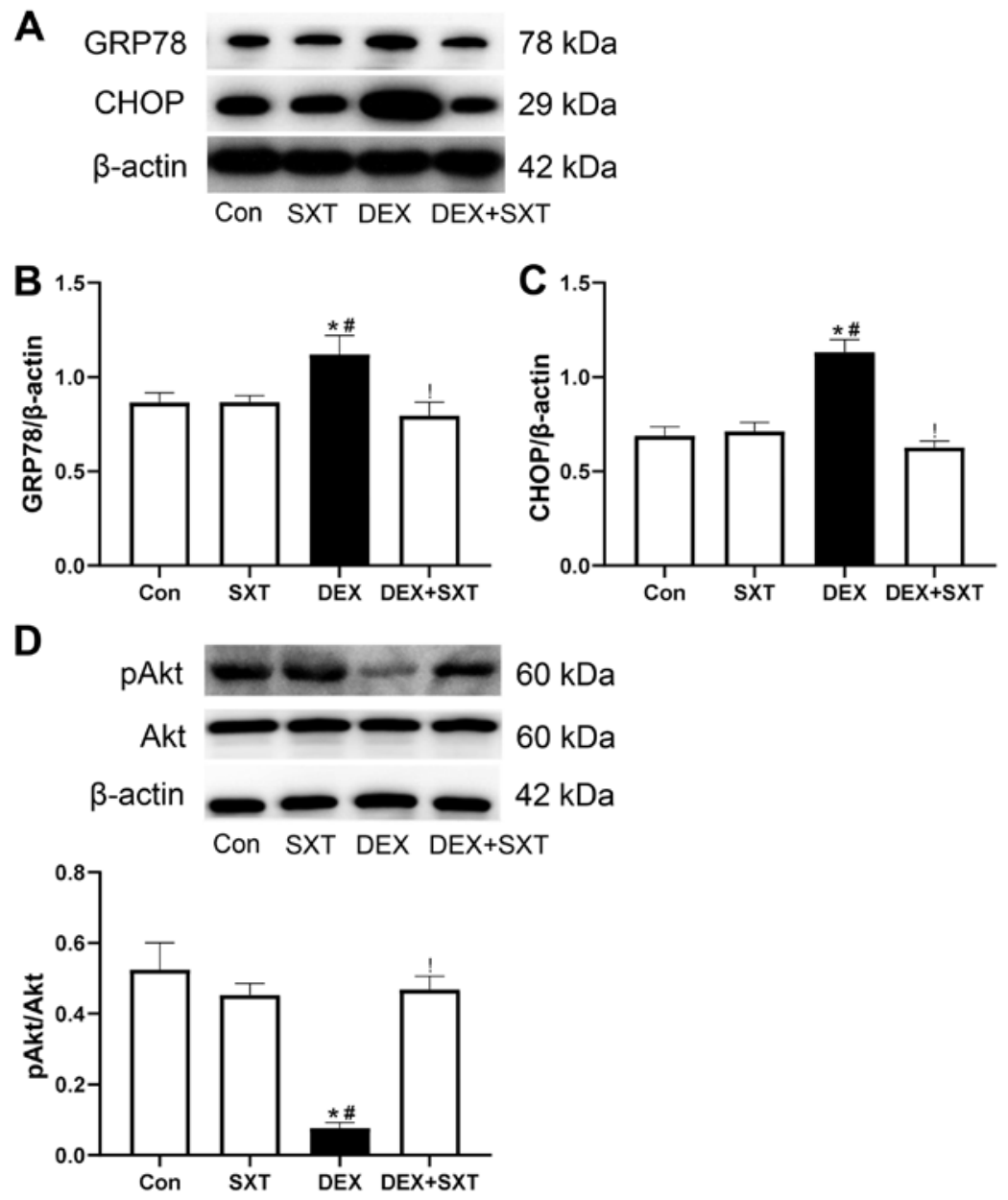

Figure 3. SXT inhibits endoplasmic reticulum stress activation and improves Akt signaling in the aorta of dexamethasone-treated rats. (A) Representative western blotting image. (B) GRP78 and (C) CHOP protein expression levels detected by western blotting. (D) pAkt and Akt protein expression levels detected by western blotting. $\mathrm{N}=8$ /group. The data are presented as the mean $\pm \mathrm{SD}$. ${ }^{*} \mathrm{P}<0.05$ vs. Con; ${ }^{*} \mathrm{P}<0.05$ vs. $\mathrm{SXT}$; $\mathrm{P}<0.05$ vs. DEX. DEX, dexamethasone; Con, control; SXT; shuxuetong; pAKT, phosphorylated Akt; GPR78, glucose-regulated protein 78; CHOP, C/EBP-homologous protein.

Increases in DEX-induced expression levels of sclerostin are attenuated by SXT. The sclerostin protein expression levels in the femoral head were detected by immunohistochemical staining (Fig. 7). The sclerostin protein expression levels in the femoral head of rats with DEX were significantly increased compared with those in the control rats, and SXT treatment attenuated the DEX-induced increase in sclerostin levels (Fig. 7). Additionally, SXT injection alone did not exhibit any observable effect on sclerostin protein levels in the femoral head compared with the control group (Fig. 7).

\section{Discussion}

The present study demonstrated that DEX treatment induced calcium deposition, ALP activation, calponin and SM $22 \alpha$ downregulation, and RUNX2, BMP2, GRP78 and CHOP upregulation in the aortic tissue of rats; all these effects were ameliorated by SXT injection. In the femoral head, SXT treatment rescued the observed structural disturbance, osteoporosis and apoptosis. Notably, sclerostin protein expression levels in the aorta and femoral head significantly increased in the DEX group compared with in the control group, but this was attenuated in the DEX + SXT group.
Although SXT injection is mainly used clinically to stimulate blood circulation (17), several studies have reported its effects on blood vessels, including angiogenesis facilitation (18), amelioration of lower extremity arteriosclerosis in patients with diabetes (33), protection of cerebral microvascular endothelial cells (20) and inhibition of inflammation (22-24). The present study demonstrated the ameliorative effect of SXT injection on VC, supported by decreased deposition of calcium in the aortic media, and the inhibition of ALP activity. Thus, the results suggested that SXT may have potential for treating $\mathrm{VC}$ in the future.

Increasing evidence has indicated an important role for ERS in VC progression; studies have demonstrated that ERS activation can exaggerate $\mathrm{VC}$ by contributing to the phenotypic VSMC differentiation from contractile-like to osteoblastic-like structures $(32,34)$. The results of the present study demonstrated that the protein expression levels of two universal ERS markers, GRP78 and CHOP $(32,34)$, were increased in DEX-treated rats, alongside decreases in calponin and SM $22 \alpha$ levels, both which are markers of contractile VSMCs, and increased RUNX2 and BMP2 levels, both of which are markers of osteoblastic VSMCs (35-37). These results indicated the contribution of ERS-induced VSMC differentiation in DEX-induced VC progression, as 
A

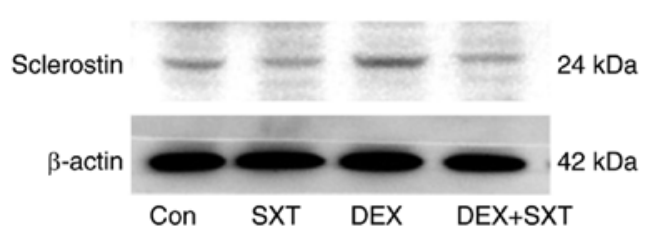

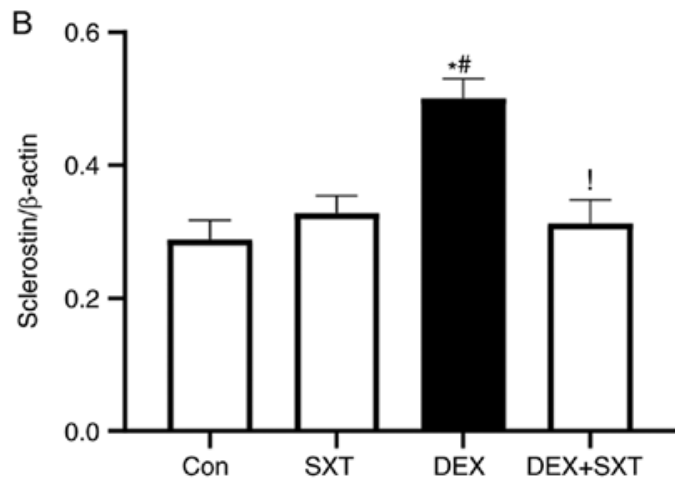

Figure 4. SXT injection inhibits the increased sclerostin levels in the aorta of dexamethasone-treated rats. (A) Representative western blotting image. (B) Sclerostin protein expression levels detected by western blotting. $\mathrm{N}=8$ /group. The data are presented as the mean $\pm \mathrm{SD}$. $\mathrm{P}<0.05$ vs. Con; ${ }^{*} \mathrm{P}<0.05$ vs. Con, ${ }^{*} \mathrm{P}<0.05$ vs. $\mathrm{SXT}$; 'P<0.05 vs. DEX. DEX, dexamethasone; Con, control; SXT; shuxuetong.

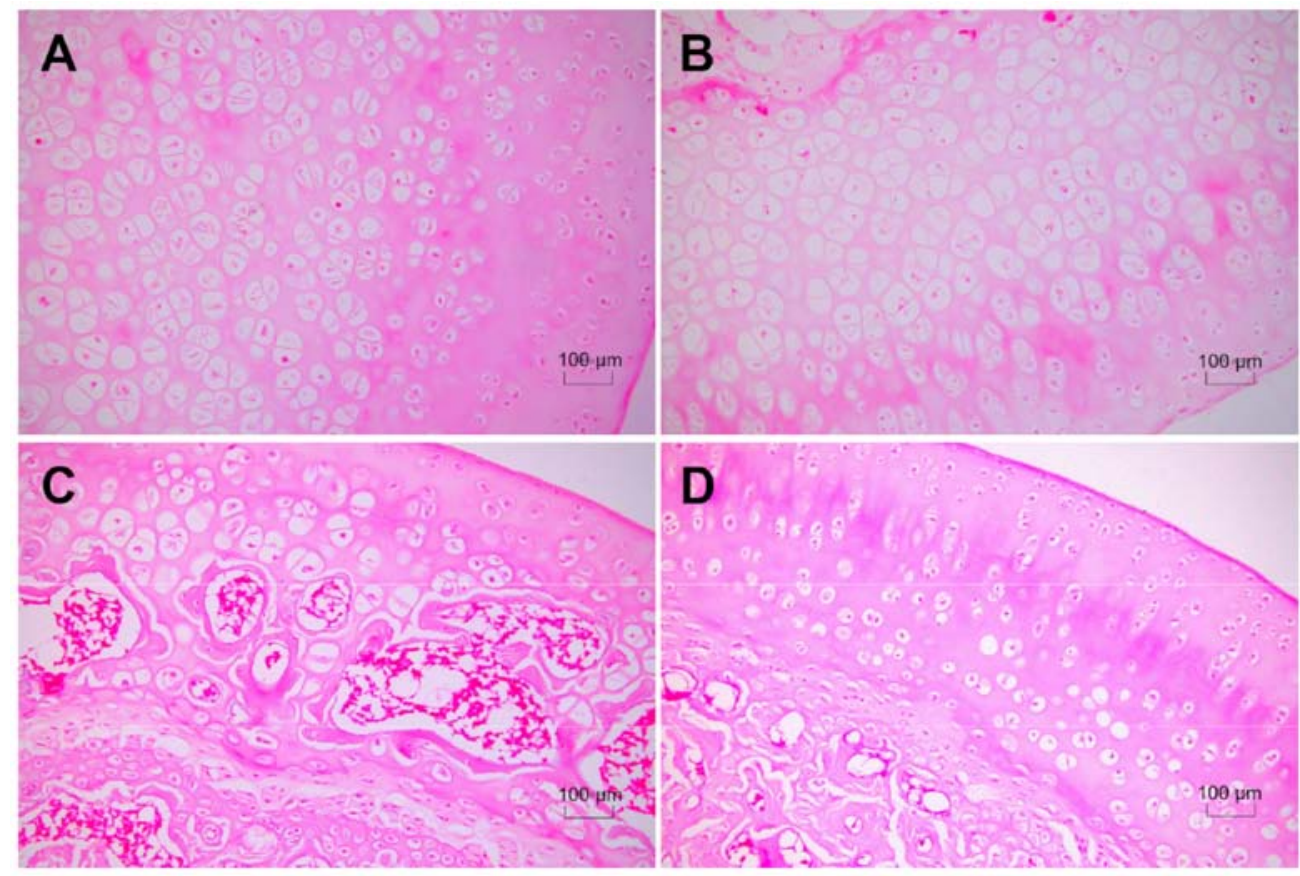

E

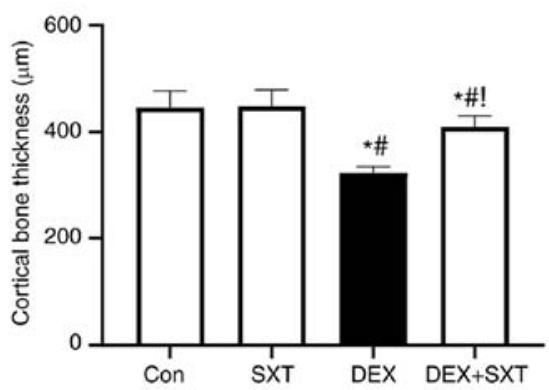

$\mathrm{F}$

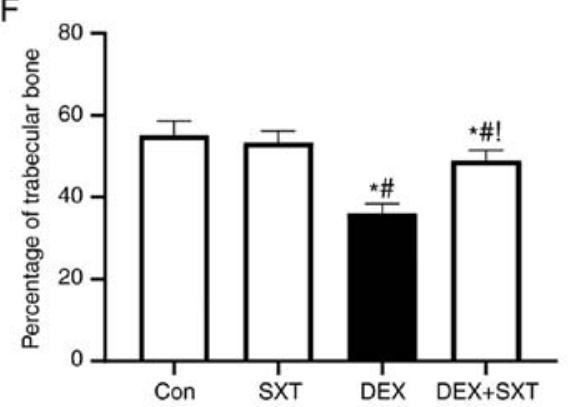

Figure 5. Hematoxylin and eosin staining of the femoral head in the (A) Con, (B) SXT, (C) DEX and (D) DEX + SXT groups. The cortical bone thickness (E) and percentage of trabecular bone $(\mathrm{F})$ were quantified. $\mathrm{N}=8$ /group. The data are presented as the mean $\pm \mathrm{SD}$. " $\mathrm{P}<0.05$ vs. Con; ${ }^{\#} \mathrm{P}<0.05$ vs. SXT; $\mathrm{P}<0.05$ vs. DEX. DEX, dexamethasone; Con, control; SXT; shuxuetong.

well as suggesting that ERS inhibition can attenuate VSMC differentiation and VC (35-37). Moreover, the present study also observed ERS inhibition, and VSMC differentiation and VC amelioration following SXT injection in DEX-treated rats. These findings suggested that SXT injection may ameliorate VC by preventing ERS-induced VSMC differentiation.
Akt may represent a link among SXT, DEX and ERS (38-40). Previous studies have demonstrated the negative effect of Akt on ERS (38-40). Activation of Akt signaling can significantly rescue ERS stimulation (38-40). Notably, Akt signaling was activated by SXT injection (19), whereas DEX prevented the activation of Akt (29). To investigate 

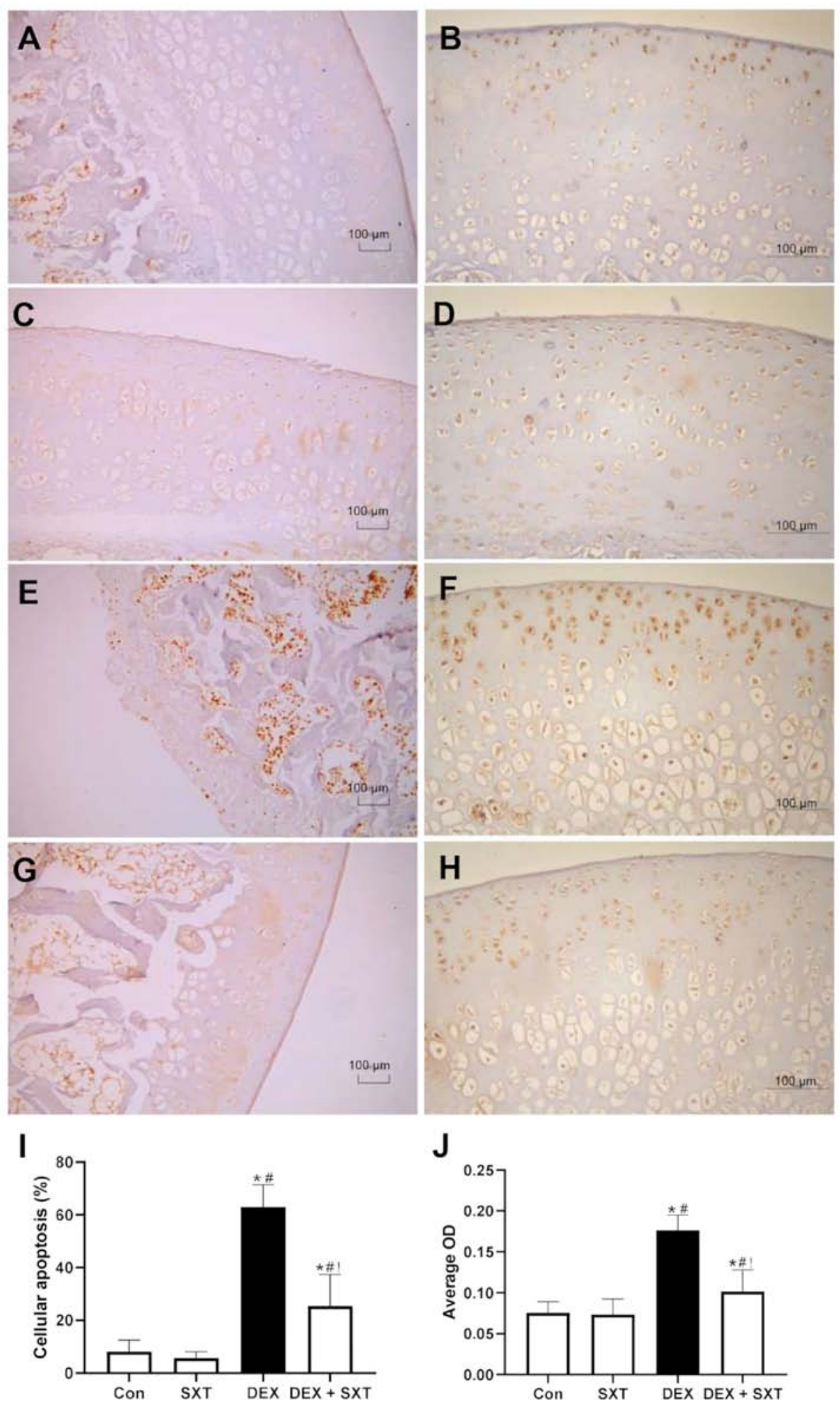

Figure 6. TUNEL staining and immunohistochemical staining of CHOP in the femoral head. Representative TUNEL staining images in the (A) Con, (C) SXT, (E) DEX and (G) DEX + SXT groups. Representative CHOP immunohistochemical staining images in the (B) Con, (D) SXT, (F) DEX and (H) DEX + SXT groups. (I) Quantitative analysis of apoptosis. (J) Quantitative analysis of CHOP levels. N=8/group. The data are presented as the mean \pm SD. "P<0.05 vs. Con; ${ }^{\#} \mathrm{P}<0.05$ vs. SXT; 'P<0.05 vs. DEX. DEX, dexamethasone; Con, control; SXT; shuxuetong; TUNEL, terminal deoxynucleotidyl transferase-mediated dUTP nick-end labeling; CHOP, C/EBP-homologous protein; OD, optical density.

whether SXT injection can activate Akt signaling, the protein expression level of pAkt was detected via western blotting. The results demonstrated that the decreased level of pAkt in DEX-treated animals was significantly rescued by SXT injection. Collectively, these findings suggested that SXT may attenuate DEX-induced ERS via activating the Akt signaling pathway.

In addition to vascular calcification, DEX can also cause osteoporosis (41-43). DEX treatment led to structural disturbance, reduction of cortical bone thickness, reduction in the 

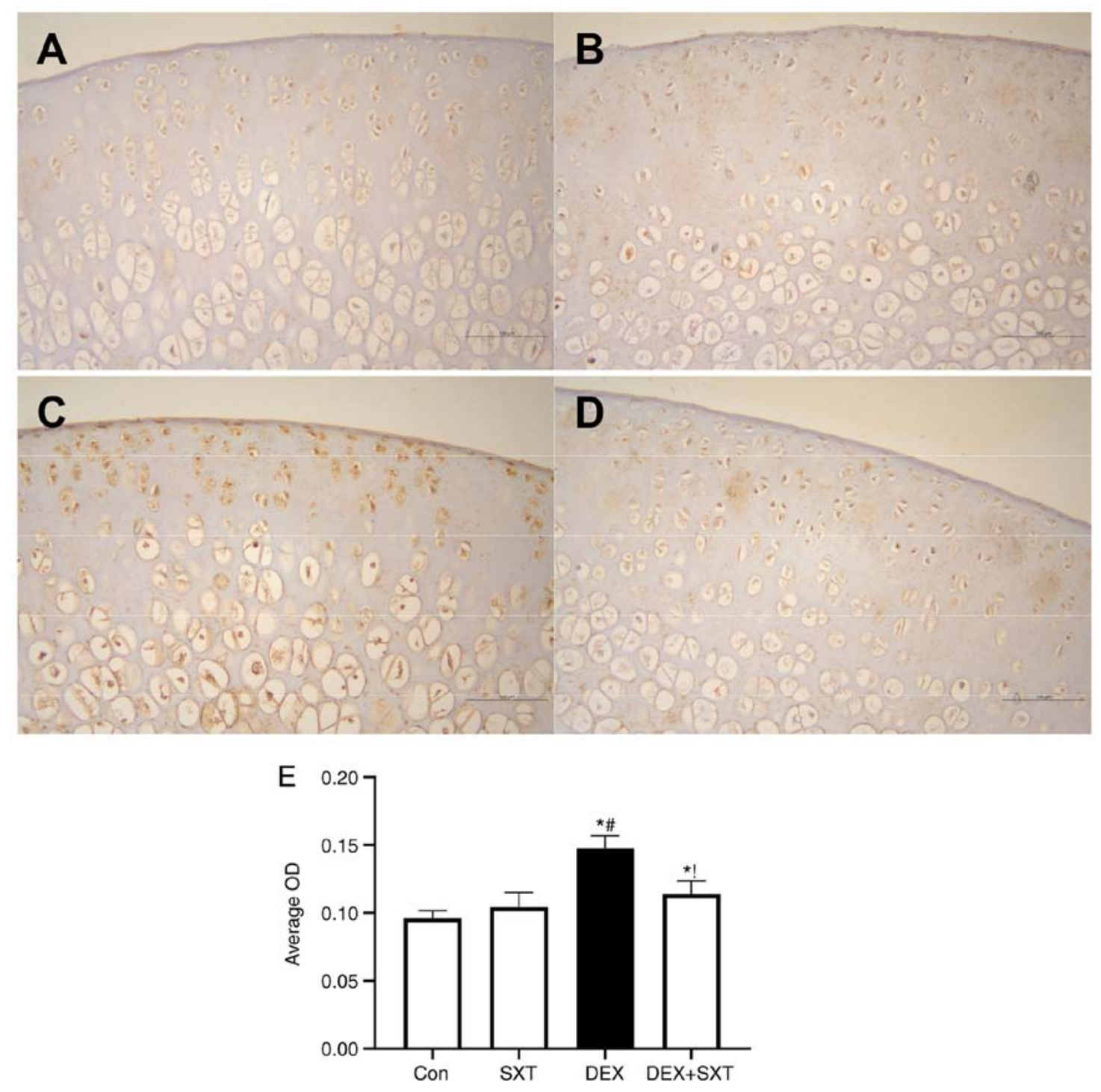

Figure 7. Immunohistochemical staining of sclerostin in the femoral head. Representative images in the (A) Con, (B) SXT, (C) DEX and (D) DEX + SXT groups. (E) Quantitative analysis of sclerostin levels. $\mathrm{N}=8$ /group. The data are presented as the mean $\pm \mathrm{SD}$. ${ }^{*} \mathrm{P}<0.05$ vs. Con; ${ }^{*} \mathrm{P}<0.05$ vs. SXT; $\mathrm{P}<0.05$ vs. DEX. DEX, dexamethasone; Con, control; SXT; shuxuetong; OD, optical density.

percentage of trabecular bone and increased cellular apoptosis in the femoral head. Notably, injection of SXT significantly ameliorated the DEX-induced femoral head impairment. Thus, the results of the present study suggested that SXT could be used to prevent and rescue the side-effects of GCs, including $\mathrm{VC}$ and osteoporosis.

To further investigate the mechanisms via which SXT ameliorated $\mathrm{VC}$ and osteoporosis, sclerostin protein expression levels in the aorta and femoral head were examined. Sclerostin was involved in both VC and osteoporosis progression $(12,44)$. Consistent with previous studies $(12,13)$, increased sclerostin protein expression levels were observed in the aortic tissue and femoral head of DEX-treated rats, and SXT injection successfully attenuated the increased sclerostin levels in both the aorta and bone. These results suggested that sclerostin inhibition may be involved in the suppressive effects of SXT on VC and osteoporosis. Unfortunately, in the present study, whether the injection of SXT ameliorated the symptoms induced by DEX by inhibiting sclerostin was not studied; the causal role of sclerostin should be fully investigated in the future.
There are two sclerostin inhibitors, romosozumab and baicalin; romosozumab is a human monoclonal antibody against sclerostin (45), and baicalin, a herb-derived flavonoid compound, binds to sclerostin via hydrophobic interactions with the amino acid residues on loop2 region, but outside the Pro-Asn-Ala-Ile-Gly motif, particularly the Arg-Gly-Lys-Trp-Trp-Arg motif (46). The beneficial effects of these two agents against osteoporosis have been reported in a number of studies (47-50). Although the effect of romosozumab on cardiovascular events including VC is controversial $(45,51)$, baicalin has been reported to relax VSMCs (52), ameliorate atherosclerosis (53), prevent trans-differentiation of VSMCs (54) and inhibit vascular remodeling (55), via which baicalin may ameliorate VC. These results suggested that inhibition of sclerostin may exert beneficial effects on both $\mathrm{VC}$ and osteoporosis.

In conclusion, the results of the present study indicated that the injection of SXT ameliorated both VC and osteoporosis, which may be mediated via regulation of sclerosis, the crosslink in the bone-vascular axis. Thus, injection of SXT may 
provide a novel strategy for the treatment of GC-induced VC and osteoporosis.

\section{Acknowledgements}

Not applicable.

\section{Funding}

This study was supported by the project for talents of Finance Department of Hebei Province (grant no. 2018133214), and the National Natural Science Foundation of China (grant no. 81770499).

\section{Availability of data and materials}

The datasets used and/or analyzed during the current study are available from the corresponding author on reasonable request.

\section{Authors' contributions}

All authors contributed to experimental design. ZX, XL, YL, HG, TH and CZ performed the experiments. ZX, XL and YL collected and analyzed the data. ZX, XL, WH and $\mathrm{XT}$ interpreted the data. WH and XT wrote and revised the manuscript. WH and XT confirm the authenticity of all the raw data. All authors read and approved the final manuscript.

\section{Ethics approval and consent to participate}

The present study was approved by the Animal Care Committee of Hebei Provincial Hospital of Chinese Medicine (Shijiazhuang, China; approval no. 2019-KY-012-01).

\section{Patient consent for publication}

Not applicable.

\section{Competing interests}

The authors declare that they have no competing interests.

\section{References}

1. Towler DA: Arteriosclerosis, bone biology, and calciotropic hormone signaling: Learning the ABCs of disease in the bone-vascular axis. J Am Soc Nephrol 26: 243-245, 2015.

2. Vassalle $C$ and Mazzone A: Bone loss and vascular calcification: A bi-directional interplay? Vascul Pharmacol 86: 77-86, 2016.

3. Tap L, Kirkham FA, Mattace-Raso F, Joly L, Rajkumar C and Benetos A: Unraveling the links underlying arterial stiffness, bone demineralization, and muscle loss. Hypertension 76: 629-639, 2020.

4. Stafford RS, Drieling RL and Hersh AL: National trends in osteoporosis visits and osteoporosis treatment, 1988-2003. Arch Intern Med 164: 1525-1530, 2004.

5. Viaene L, Behets GJ, Heye S, Claes K, Monbaliu D, Pirenne J, D'Haese PC and Evenepoel P: Inflammation and the bone-vascular axis in end-stage renal disease. Osteoporos Int 27: 489-497, 2016.

6. Zoppellaro G, Faggin E, Puato M, Pauletto P and Rattazzi M: Fibroblast growth factor 23 and the bone-vascular axis: Lessons learned from animal studies. Am J Kidney Dis 59: 135-144, 2012.
7. Kim JM, Lee WS and Kim J: Therapeutic strategy for atherosclerosis based on bone-vascular axis hypothesis. Pharmacol Ther 206: 107436, 2020.

8. Brandenburg VM, D'Haese P, Deck A, Mekahli D, Meijers B, Neven E and Evenepoel P: From skeletal to cardiovascular disease in 12 steps-the evolution of sclerostin as a major player in CKD-MBD. Pediatr Nephrol 31: 195-206, 2016.

9. Fadini GP, Rattazzi M, Matsumoto T, Asahara T and Khosla S: Emerging role of circulating calcifying cells in the bone-vascular axis. Circulation 125: 2772-2781, 2012.

10. Pieralice S, Vigevano F, Del Toro R, Napoli N and Maddaloni E: Lifestyle management of diabetes: Implications for the bone-vascular axis. Curr Diab Rep 18: 84, 2018.

11. D'Onofrio L, Maddaloni E and Buzzetti R: Osteocalcin and sclerostin: Background characters or main actors in cardiovascular disease? Diabetes Metab Res Rev 36: e3217, 2020.

12. Figurek A, Rroji $M$ and Spasovski G: Sclerostin: A new biomarker of CKD-MBD. Int Urol Nephrol 52: 107-113, 2020.

13. De Maré A, Maudsley S, Azmi A, Hendrickx JO, Opdebeeck B, Neven E, D'Haese PC and Verhulst A: Sclerostin as regulatory molecule in vascular media calcification and the Bone-Vascular Axis. Toxins (Basel) 11: 428, 2019.

14. Leto G, D'Onofrio L, Lucantoni F, Zampetti S, Campagna G, Foffi C, Moretti C, Carlone A, Palermo A, Leopizzi M, et al: Sclerostin is expressed in the atherosclerotic plaques of patients who undergoing carotid endarterectomy. Diabetes Metab Res Rev 35: e3069, 2019.

15. Morales-Santana S, García-Fontana B, García-Martín A, Rozas-Moreno P, García-Salcedo JA, Reyes-García R and Muñoz-Torres M: Atherosclerotic disease in type 2 diabetes is associated with an increase in sclerostin levels. Diabetes Care 36: 1667-1674, 2013.

16. Saag KG, Petersen J, Brandi ML, Karaplis AC, Lorentzon M, Thomas T, Maddox J, Fan M, Meisner PD and Grauer A: Romosozumab or alendronate for fracture prevention in women with osteoporosis. N Engl J Med 377: 1417-1427, 2017.

17. Cai L, Huang W and Lin D: Effects of traditional Chinese medicine Shuxuetong injection on random skin flap survival in rats. ScientificWorldJournal 2014: 816545, 2014.

18. Jin X, Shen G, Gao F, Zheng X, Xu X, Shen F, Li G, Gong J, Wen L, Yang X and Bie X: Traditional Chinese drug ShuXueTong facilitates angiogenesis during wound healing following traumatic brain injury. J Ethnopharmacol 117: 473-477, 2008.

19. Liu X, Qing Wang, Cui Y, Li X and Yang H: In-depth transcriptomic and proteomic analyses of the hippocampus and cortex in a rat model after cerebral ischemic injury and repair by Shuxuetong (SXT) injection. J Ethnopharmacol 249: 112362, 2020.

20. Sun ZY, Wang FJ, Guo H, Chen L, Chai LJ, Li RL, Hu LM, Wang $\mathrm{H}$ and Wang SX: Shuxuetong injection protects cerebral microvascular endothelial cells against oxygen-glucose deprivation reperfusion. Neural Regen Res 14: 783-793, 2019.

21. Zhang X, Xiao B and Hu CL: Study in the mechanism of Shuxuetong injection on antithrombosis and thrombolysis. Zhongguo Zhong Yao Za Zhi 30: 1950-1952, 2005.

22. Chen X, Ding X, Liu L, Zhao L, Jiang Z and Wang X: Effect of Shuxuetong injection on reducing expression of vascular adhesion molecules in cerebral ischemia and reperfusion rats. J New Chin Med 45: 128-130, 2013.

23. Wei J, Chen B, Tian Z, Luo K and Deng W: Effect of Shuxuetong injection on VEGF expression of hind-limb ischemic vascular disorder in diabetic rats. Zhong Xi Yi Jie He Xin Nao Xue Guan Bing Za Zhi 7: 1048-1049, 2009 (In Chinese).

24. Zhang Y, Zhou S and Wang B: Effect of Shuxuetong injection on ET-1, sICAM-1, TNF- $\alpha$ and P-selection in diabetes mellitus with coronary artery disease. Zhong Xi Yi Jie He Xin Nao Xue Guan Bing Za Zhi 6: 1271-1272, 2008 (In Chinese).

25. Xi L, Song Y, Wu W, Qu Z, Wen J, Liao B, Tao R, Ge J and Fang D: Investigation of bone matrix composition, architecture and mechanical properties reflect structure-function relationship of cortical bone in glucocorticoid induced osteoporosis. Bone 136: 115334, 2020.

26. Ren H, Liang D, Jiang X, Tang J, Cui J, Wei Q, Zhang S, Yao Z, Shen $G$ and Lin S: Variance of spinal osteoporosis induced by dexamethasone and methylprednisolone and its associated mechanism. Steroids 102: 65-75, 2015.

27. Mori K, Shioi A, Jono S, Nishizawa Y and Morii $\mathrm{H}$ : Dexamethasone enhances In vitro vascular calcification by promoting osteoblastic differentiation of vascular smooth muscle cells. Arterioscler Thromb Vasc Biol 19: 2112-2118, 1999. 
28. Kirton JP, Wilkinson FL, Canfield AE and Alexander MY: Dexamethasone downregulates calcification-inhibitor molecules and accelerates osteogenic differentiation of vascular pericytes: Implications for vascular calcification. Circ Res 98: 1264-1272, 2006.

29. Tao SC, Yuan T, Rui BY, Zhu ZZ, Guo SC and Zhang CQ: Exosomes derived from human platelet-rich plasma prevent apoptosis induced by glucocorticoid-associated endoplasmic reticulum stress in rat osteonecrosis of the femoral head via the Akt/Bad/Bcl-2 signal pathway. Theranostics 7: 733-750, 2017.

30. China Animal Management Rule, documentation No. 55, 2001, the Ministry of Health of P.R. China.

31. The National Institutes of Health Guide for the Care and Use of Laboratory Animals, NIH Publications no. 8023, revised 1978.

32. Duan X, Zhou Y, Teng X, Tang C and Qi Y: Endoplasmic reticulum stress-mediated apoptosis is activated in vascular calcification. Biochem Biophys Res Commun 387: 694-699, 2009.

33. Feng K, Tan J and Chen Y: Treatment of lower extremity diabetic atherosclerotic obliterans with Shuxuetong injection. Zhongguo Zhong Xi Yi Jie He Za Zhi 29: 255-257, 2009 (In Chinese).

34. Duan XH, Chang JR, Zhang J, Zhang BH, Li YL, Teng X, Zhu Y, Du J, Tang CS and Qi YF: Activating transcription factor 4 is involved in endoplasmic reticulum stress-mediated apoptosis contributing to vascular calcification. Apoptosis 18: 1132-1144, 2013.

35. Chang JR, Duan XH, Zhang BH, Teng X, Zhou YB, Liu Y, Yu YR, Zhu Y, Tang CS and Qi YF: Intermedin1-53 attenuates vascular smooth muscle cell calcification by inhibiting endoplasmic reticulum stress via cyclic adenosine monophosphate/ protein kinase A pathway. Exp Biol Med (Maywood) 238 : 1136-1146, 2013.

36. Yang R, Teng X, Li H, Xue HM, Guo Q, Xiao L and Wu YM: Hydrogen sulfide improves vascular calcification in rats by inhibiting endoplasmic reticulum stress. Oxid Med Cell Longev 2016: $9095242,2016$.

37. Hao W, Yang R, Yang Y, Jin S, Li Y, Yuan F, Guo Q, Xiao L, Wang X, Wang F, et al: Stellate ganglion block ameliorates vascular calcification by inhibiting endoplasmic reticulum stress. Life Sci 193: 1-8, 2018

38. Teng X, Song J, Zhang G, Cai Y, Yuan F, Du J, Tang C and Qi Y: Inhibition of endoplasmic reticulum stress by intermedin(1-53) protects against myocardial injury through a PI3 kinase-Akt signaling pathway. J Mol Med (Berl) 89: 1195-1205, 2011.

39. Tian T, Zhao Y, Nakajima S, Huang T, Yao J, Paton AW, Paton JC and Kitamura M: Cytoprotective roles of ERK and Akt in endoplasmic reticulum stress triggered by subtilase cytotoxin. Biochem Biophys Res Commun 410: 852-858, 2011.

40. Liu H, Li X, Qin F and Huang K: Selenium suppresses oxidative-stress-enhanced vascular smooth muscle cell calcification by inhibiting the activation of the PI3K/AKT and ERK signaling pathways and endoplasmic reticulum stress. J Biol Inorg Chem 19: 375-388, 2014.

41. Rathi A, Ishaq M, Najmi AK and Akhtar M: Trigonelline demonstrated ameliorative effects in dexamethasone induced osteoporotic rats. Drug Res (Stuttg) 70: 257-264, 2020.

42. Qin Z, Li S, Zhang X, Liu G, Gu M, Zhang N, Liu J, Ji Z, Li K, Han Y and Zhai H: Combination therapy of wuweizi (Schisandrae Chinensis Fructus) and dexamethasone alleviated dexamethasone-induced glucocorticoid osteoporosis in rats with idiopathic pulmonary fibrosis. Biomed Res Int 2020: 6301697, 2020.
43. Fu L, Wu W, Sun X and Zhang P: Glucocorticoids enhanced osteoclast autophagy through the PI3K/Akt/mTOR signaling pathway. Calcif Tissue Int 107: 60-71, 2020.

44. Pietrzyk B, Smertka M and Chudek J: Sclerostin: Intracellular mechanisms of action and its role in the pathogenesis of skeletal and vascular disorders. Adv Clin Exp Med 26: 1283-1291, 2017.

45. Asadipooya K and Weinstock A: Cardiovascular outcomes of romosozumab and protective role of alendronate. Arterioscler Thromb Vasc Biol 39: 1343-1350, 2019.

46. Alazmi M and Motwalli O: In silico virtual screening, characterization, docking and molecular dynamics studies of crucial SARS-CoV-2 proteins. J Biomol Struct Dyn: Aug 7, 2020 (Epub ahead of print). doi: 10.1080/07391102.2020.1803965.

47. Khosla S: Bone diseases: Romosozumab-on track or derailed? Nat Rev Endocrinol 13: 697-698, 2017.

48. Fontalis A, Kenanidis E, Kotronias RA, Papachristou A, Anagnostis P, Potoupnis M and Tsiridis E: Current and emerging osteoporosis pharmacotherapy for women: State of the art therapies for preventing bone loss. Expert Opin Pharmacother 20: 1123-1134, 2019.

49. Chen Z, Pan X, Sheng Z, Yan G, Chen L and Ma G: Baicalin suppresses the proliferation and migration of Ox-LDL-VSMCs in atherosclerosis through upregulating miR-126-5p. Biol Pharm Bull 42: 1517-1523, 2019.

50. Lu X, He W, Yang W, Li J, Han W, Liu Q, Zhang T, Jiang J, Qin A and Qian Y: Dual effects of baicalin on osteoclast differentiation and bone resorption. J Cell Mol Med 22: 5029-5039, 2018.

51. Turk JR, Deaton AM, Yin J, Stolina M, Felx M, Boyd G, Bienvenu JG, Varela A, Guillot M, Holdsworth G, et al: Nonclinical cardiovascular safety evaluation of romosozumab, an inhibitor of sclerostin for the treatment of osteoporosis in postmenopausal women at high risk of fracture. Regul Toxicol Pharmacol 115: 104697, 2020.

52. Ding L, Jia C, Zhang Y, Wang W, Zhu W, Chen Y and Zhang T: Baicalin relaxes vascular smooth muscle and lowers blood pressure in spontaneously hypertensive rats. Biomed Pharmacother 111: 325-330, 2019.

53. Liu Y, Jia L, Min D, Xu Y, Zhu J and Sun Z: Baicalin inhibits proliferation and promotes apoptosis of vascular smooth muscle cells by regulating the MEG3/p53 pathway following treatment with ox-LDL. Int J Mol Med 43: 901-913, 2019.

54. Lv P, Zhang F, Yin YJ, Wang YC, Gao M, Xie XL, Zhao LL, Dong LH, Lin YL, Shu YN, et al: SM22 $\alpha$ inhibits lamellipodium formation and migration via Ras-Arp2/3 signaling in synthetic VSMCs. Am J Physiol Cell Physiol 311: C758-C767, 2016.

55. Dong LH, Wen JK, Miao SB, JiaZ, Hu HJ, Sun RH, Wu Y and Han M: Baicalin inhibits PDGF-BB-stimulated vascular smooth muscle cell proliferation through suppressing PDGFR $\beta$-ERK signaling and increase in p27 accumulation and prevents injury-induced neointimal hyperplasia. Cell Res 20: 1252-1262, 2010.

This work is licensed under a Creative Commons Attribution-NonCommercial-NoDerivatives 4.0 International (CC BY-NC-ND 4.0) License. 